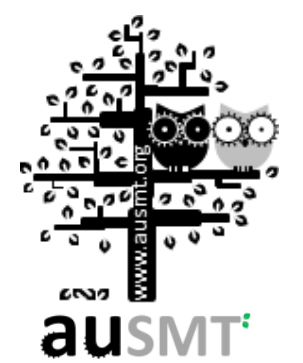

\title{
An Integrated Microfluidic Chip for Rapid Methanol Detection
}

\section{Ting-Fu Hong1, Wei-Jhong Ju², Chien-Hsiung Tsai ${ }^{3}$, Yao-Nan Wang ${ }^{3}$, , and Lung-Ming $\mathrm{Fu}^{1}$, *}

${ }^{1}$ Department of Materials Engineering, National Pingtung University of Science and Technology, Taiwan

${ }^{2}$ Department of Engineering Science, National Cheng Kung University, Taiwan

${ }^{3}$ Department of Vehicle Engineering, National Pingtung University of Science and Technology, Taiwan

(Received 31 October 2011; Accepted 9 January 2012; Published on line 1 March 2012)

*Corresponding author: ynwang@npust.edu.tw (Yao-Nan Wang); loudyfu@mail.npust.edu.tw (Lung-Ming Fu)

DOI: $10.5875 /$ ausmt.v2i1.112

\begin{abstract}
A}$ widely-available $\mathrm{CO}_{2}$ laser scriber is used to perform direct-writing ablation on a poly(methyl methacrylate) (PMMA) substrate to create a microfluidic chip for the rapid detection of methanol. The microfluidic designs are created using commercial layout software and are converted into the command signals required to drive the laser scriber in such a way as to reproduce the desired microchannel configuration on the surface of a PMMA substrate. Experimental results indicate that, using the proposed integrated microfluidic chip, linearity expression $\mathrm{R}^{2}$ can reach 0.9972 when using 2 unit methanol oxidase (MOX) and basic fuchsin to detect methanol. The proposed device is thus a valuable tool for rapid methanol detection, with its micro mixer system providing a simple yet effective solution for mixing problems in the field of micro-total-analysis-systems.
\end{abstract}

Keywords: Methanol; $\mathrm{CO}_{2}$ laser; microfluidic chip; PMMA

\section{Introduction}

In recent decades, microsystem technologies have attracted significant interest among microfluidics researchers for the development of biochemical applications in food safety inspection and quality control $[1,2]$, drug discovery [3-5], environmental monitoring [6, 7], and biomedicines [8-10]. Several functional microfluidic devices have been developed to perform a variety of tasks, including sample pre-treatment and injection, species mixing, polymerase chain reaction, and cell/particle separation and counting [11-17]. Microfluidic devices present numerous crucial advantages, including reduced sample and reagent use, enhanced efficiency, improved sensitivity, shorter processing times, lower power consumption, greater portability, lower fabrication and operating costs, and the potential for integration with other miniaturized devices.

Recently, numerous groups have investigated methods for integrated microfluidic devices [18-20] for a wide range of applications in chemical and biological analysis. Lin et al [21] presented a rapid microfluidic mixer using a freeze-quenching technique, providing a useful means of trapping meta-stable intermediates populated during rapid chemical or biochemical reactions. $\mathrm{Fu}$ and $\mathrm{Lin}$ [22] presented a novel DNA digestion system using periodic electrokinetic driving forces and a high performance microfluidic mixer.

Methanol poisoning is usually caused by incorrect use of wood alcohol. However, other types of alcohol contain even higher concentrations of methanol (e.g., general alcohol and that found in fruit wine). According to Taiwan's Department of Alcoholic Beverage Control Standards, the methanol content in general alcohol is $1000 \mathrm{ppm}$, while wine contains $3000 \mathrm{ppm}$ (in terms of pure ethanol). Current common methanol detection methods include gas chromatography detection (GC) and 
potassium permanganate oxidation (commonly referred to as the traditional method) $[23,24]$. While GC detection is fast and highly accurate, but required equipment and materials are expensive, making it unsuitable for use in testing devices for the general population or small businesses. The traditional test method, meanwhile, suffers from low accuracy due to the dissimilar reactive property that non-specific potassium permanganate oxidation has on methanol versus distilled alcohol. Some researchers have used methanol oxidase (MOX) in place of non-specific potassium permanganate to form formaldehyde via the methanol oxidation process, followed by mixing with basic fuchsin for colorimetry [25, 26].

Washing and drying testing equipment is a time-consuming process, while quartz glass for sample testing in spectrophotometers is expensive. This study presents a fast, cost-effective and microfluidic method for disposable methanol detection applications. The detection process is simple: a micro syringe-pump injects methanol and MOX into the microchip which is allowed to stand momentarily; magenta $\& \mathrm{HCl}$ are added and the entire microchip is directly inserted into the spectrophotometer. The present study used a $\mathrm{CO}_{2}$ laser system to ablate PMMA substrates, using a defocusing

\footnotetext{
Ting-Fu Hong graduated in Mechanical Engineering from the National Cheng-Kung University (Taiwan) in 1996. He was awarded M.S. degree in Metallic and Ceramic Materials from the University of Manchester Institute of Science and Technology (UMIST, UK) in 2001 and Ph.D. in Materials Science from the University of London (UK) in 2006. His graduate studies were focused on the characterisation techniques of porous materials and the development of biomaterials. He is currently an assistant professor in the Department of Materials Engineering at National Pingtung University of Science and Technology. His research interests are in functional surface treatments on metallic biomaterials, synthesis of micro-nano metallic and ceramic particles, and MEMS fabrication technologies.
}

Wei-Jhong Ju received M.S. degree in Materials Engineering from National Pingtung University of Science and Technology (NPUST), Taiwan, in 2009. He is currently a doctoral candidate in Engineering Science at National Cheng Kung University (NCKU), where he is developing integration of microdevices for detecting food additive.

Chien-Hsiung Tsai received M.S. and Ph.D. degrees from the Department of Mechanical Engineering from National Taiwan University (NTU), Taiwan, in 1994. He is currently a Professor in the Department of Vehicle Engineering at National Pingtung University of Science and Technology. His current research involves thermo-fluid engineering and industrial hydrodynamics.

Yao-Nan Wang received M.S. and Ph.D. degrees from the Department of Mechanical Engineering from National Cheng Kung University (NCKU), Taiwan, in 2003 and 2008, respectively. He is currently an Assistant Professor in the Department of Vehicle Engineering at National Pingtung University of Science and Technology. His current research involves thermo-fluid engineering and integration of microdevices.

Lung-Ming Fu received M.S. and Ph.D. degrees in Engineering Science from National Cheng Kung University (NCKU), Taiwan, in 1997 and 2001. He had his postdoc training in Department of Engineering Science at NCKU during 2002-2003. He is currently a professor in the Graduate Institute of Materials Engineering at National Pingtung University of Science and Technology. His research interests are in Microfluidic systems, MEMS fabrication technologies, Micro-sensor and Computational fluid dynamics.

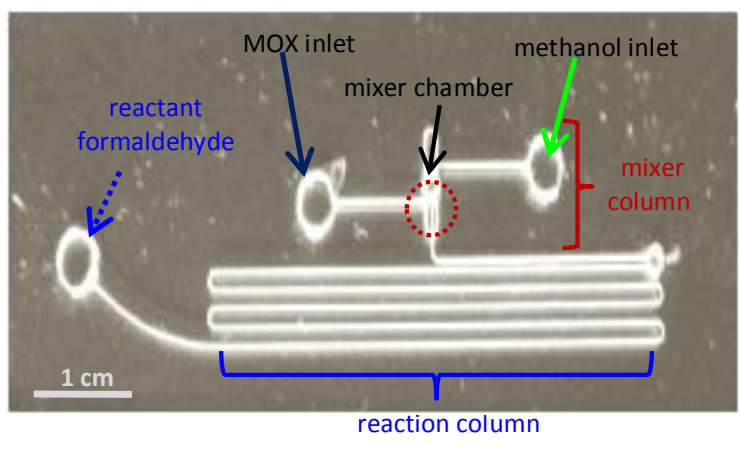

(a)

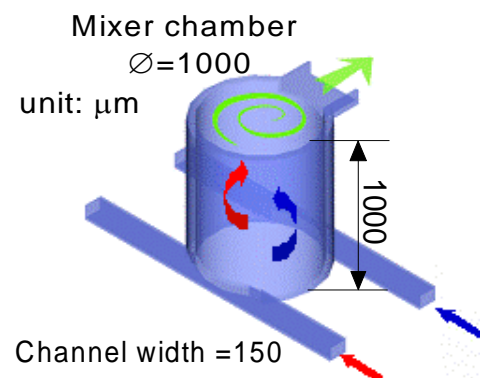

(b)

Figure 1. (a) photograph of methanol rapid detection microfluidic chip, and (b) schematic illustration of micro mixer-chamber geometry.

laser beam method to fabricate the integrated microfluidic chip. Figure 1 presents a schematic illustration and a photograph of the rapid detection of methanol using the integrated microfluidic chip, which features three entrances for the injection of methanol, enzyme, magenta, and $\mathrm{HCl}$. All of the reagents were mixed and reacted in the rectangular reservoir, and then tested using the UV spectrophotometer. Experimental results indicate that the linear expression $R^{2}$ reaches 0.9972 using the proposed integrated microfluidic chip, as opposed to 0.9985 with the traditional method using the mixture of two-unit methanol oxidase (MOX) and basic fuchsin (BF, Schiff method) for the detection of various concentrations of methanol.

\section{Experimental setup}

Figure 2 presents the experimental setup for PMMA microchannel fabrication. The $\mathrm{CO}_{2}$ laser (V-12, Laser Pro Venus laser system) has a maximum output power of $12 \mathrm{~W}$, an output beam diameter of $3.5 \mathrm{~mm}$ at the aperture, a beam divergence (full angle) of $4 \mathrm{mrad}$, and a wavelength of $10.6 \mu \mathrm{m}$. The beam scanning speed was programmable over a range of 5 to $500 \mathrm{~mm} / \mathrm{s}$. The sample was mounted on a $300 \times 210 \mathrm{~mm}^{2} \mathrm{X}-\mathrm{Y}$ plane workspace driven by a DC servo control system. The substrate was secured on a platform that can move in the Z-axis. The microfluidic pattern was designed using commercially-available computer software (CorelDraw 


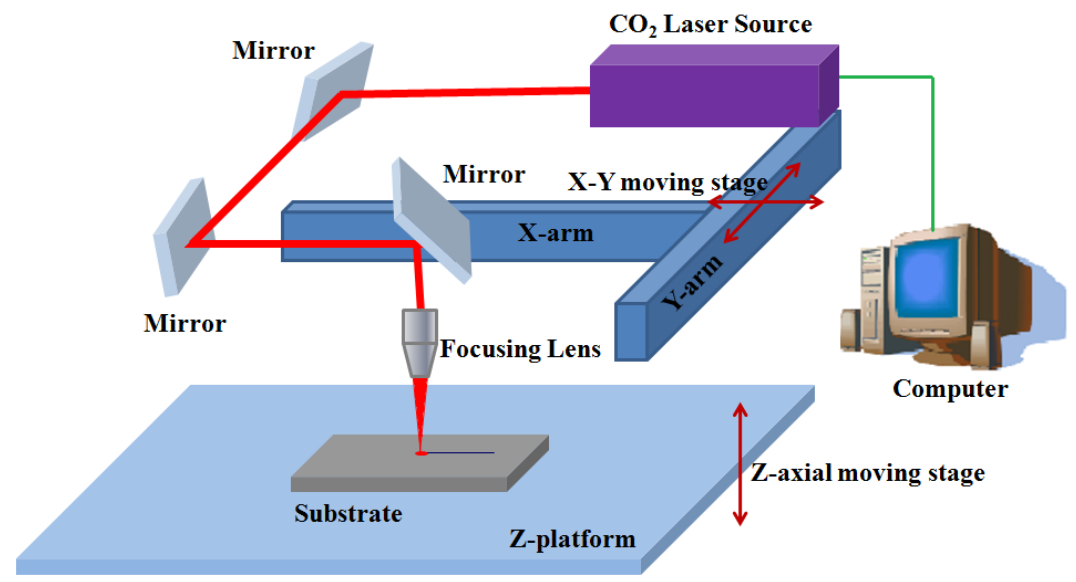

Figure 2. Schematic illustration of $\mathrm{CO}_{2}$ laser system used to pattern PMMA substrates.

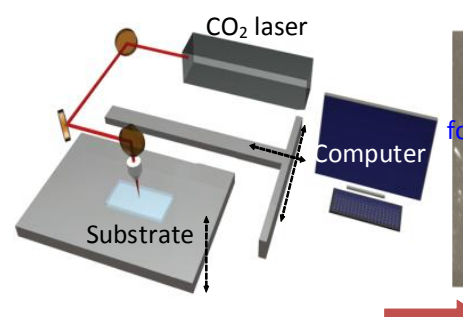

(a)

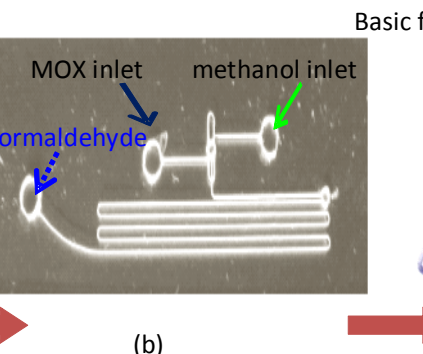

(b)

Design and fabrication

Basic fuchsin formaldehyde

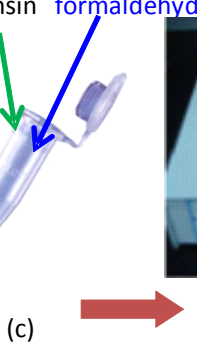

(c)

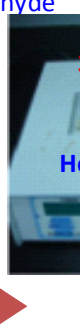

Heating



(e)

Detection

Figure 3. Schematic illustration showing major steps in chip fabrication and methanol concentration detection procedures: (a) design and fabrication; (b) chip and sample injection; (c) Eppendrof tube; (d) heating; (e) detection.

and AutoCAD). It was then translated into computer-controlled motion while the laser beam was used to etch the substrate. In the experiments, the laser power was set between 1.2 and $6 \mathrm{~W}$, and the scanning speed varied between 50 and $300 \mathrm{~mm} / \mathrm{s}$. Experiments were focused on fabricated channels at low laser intensity using the laser beam defocusing method (the defocused laser beam ablation method is described in detail in [27]). The resulting channel widths ranged from 100 to $200 \mu \mathrm{m}$, while the channel depths ranged from 30 to $300 \mu \mathrm{m}$. Meanwhile, the upper substrate of the microfluidic device was formed by drilling holes using the $\mathrm{CO}_{2}$ laser etched microscope slide. Following micromachining, the two plates were joined using a thermal bonding process performed in a hot embossing machine at a temperature of $105^{\circ} \mathrm{C}$ for 20 minutes and a pressure of $5 \mathrm{~kg} / \mathrm{cm}^{2}$.

Figure 3 presents the experimental procedure steps of the method for integrated rapid microfluidic detection of methanol concentrations. The sample reagents included methanol, alcohol oxidase, basic fuchsin, $\mathrm{HCl}, \mathrm{PBS}$, and DI water. Reagent preparation entailed: (1) methanol concentrations ranging from 10 30 ppm prepared by mixing methanol and DI water in appropriate quantities; (2) $67.5 \mathrm{ml}$ of PBS and $100 \mu \mathrm{l}$ alcohol oxidase mixed into two units; (3) $0.5 \mathrm{~g}$ basic fuchsin and $100^{\circ} \mathrm{C}$ DI water mixed with heating and stirring, followed by $1.5 \mathrm{~g}$ of anhydrous sodium sulfite dissolved in $10 \mathrm{ml} \mathrm{DI}$ water and mixed with fuchsin, along with $1 \mathrm{ml}$ of sulfuric acid in a concentration of $36 \mathrm{~N}$ and $10 \mathrm{ml}$ hydrochloric acid in a concentration of $36 \mathrm{~N}$, cooled to room temperature and then diluted to $100 \mathrm{ml}$; and (4) diluted $\mathrm{HCl}$ in a concentration of $36 \mathrm{~N}$ to $1 \mathrm{~N}$. In this experiment, the microchannel, micromixer, reservoir on the PMMA microchip, and substrate cutting were fabricated using $\mathrm{CO}_{2}$ laser machining processes and sealed by a hot-press bonding technique (see Figure 3(a), 3(b)). In Figure 3(c), a methanol detection chip is installed in the experimental platform to achieve a reaction. The reactant of formaldehyde was then inserted into the eppendrof tube and mixed with basic fuchsin. Methanol and MOX were injected into the microchip using a syringe pump, mixed in an ultrasonic cleaner for $5 \mathrm{~min}$ and then maintained at a constant $25^{\circ} \mathrm{C}$ for approximately $25 \mathrm{~min}$. The basic fuchsin and $\mathrm{HCl}$ were then increased in the microchip and maintained at a constant $35^{\circ} \mathrm{C}$ (see Figure $3(\mathrm{~d})$ ). The vortex effect enables MOX and methanol to become a uniform mixture in the micromixer, and MOX reacts with methanol to form formaldehyde, which can be written as 


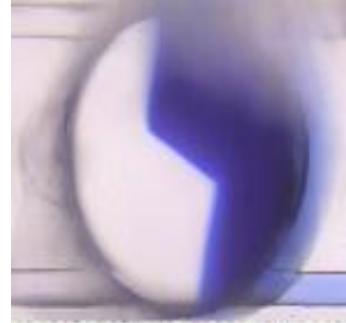

(a)

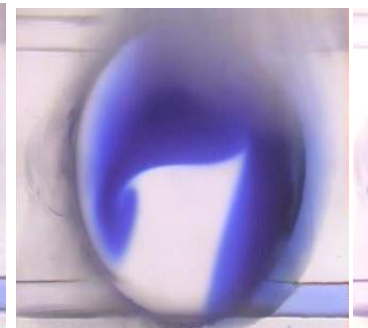

(b)

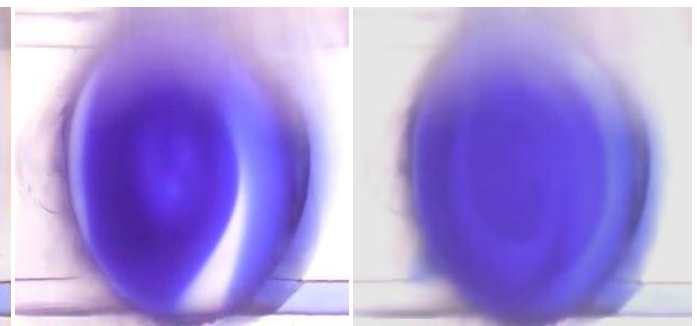

(c) (d)

Figure 4. Experimental images showing flow rotation effect induced in the micro-mixer's micro-chamber at Re = 4 after (a) $2 \mathrm{~s}$, (b) $4 \mathrm{~s}$, (c) $7 \mathrm{~s}$, and (d) $10 \mathrm{~s}$.

$\mathrm{CH}_{3} \mathrm{OH}+\mathrm{O}_{2} \stackrel{\text { MOX }}{\longrightarrow} \mathrm{HCOH}+\mathrm{H}_{2} \mathrm{O}_{2}$. The syringe pump was then turned off and the microfluidic chip was removed then turned off and the microfluidic chip was removed from the experimental platform. The detection chip was then inserted into a spectrophotometer (Model U-2000, Tokyo, Japan) to observe the corresponding absorption spectrum (see Figure 3(e)).

\section{Results and discussion}

In the micro reaction experiment, a microscope (E-400, Nikon, Japan) and image capture card (DVD PKB, V-gear, Taiwan) were used to capture images to determine mixing efficiency in the chip's mixing chamber. To generate a high-efficiency mixing effect from the vortex in the micro-mixer, syringe pumps were used to inject a solution with blue dye and DI water at an optimal flow rate.

Figure 4 presents a series of experimental images showing the evolution of the flow rotation effect within the circular microchamber with blue dye injected into the micro-mixer with a Reynolds number of $\operatorname{Re}=4$. Detailed descriptions of the mixing effect and mixing mechanisms of circular microfluidic mixers can be found in [28]. The images show that, when the DI water and a solution containing blue dye are initially loaded into the microchamber, the species concentration distribution exhibits two distinct regions. However, the driving force is sufficient to establish a rotational flow effect in the microchamber, and thus a 3-D vortex is formed within 10 seconds (see Figure 4(d)). Analysis of the outlet region results shows that a mixing ratio of $95.8 \%$ is obtained for a Reynolds number of $\operatorname{Re}=4$. Figure 5 shows that the vortex structure results in an effective mixing of the two species at even low Reynolds number values. Moreover, the mixing performance is found to improve as the Reynolds number increases due to the corresponding increase in the intensity of the vortex structure. Overall, the results show that the optimal mixing ratio ( 95\%) is obtained at a Reynolds number greater than or equal to 4, which was used as the value for all remaining experiments and simulations.

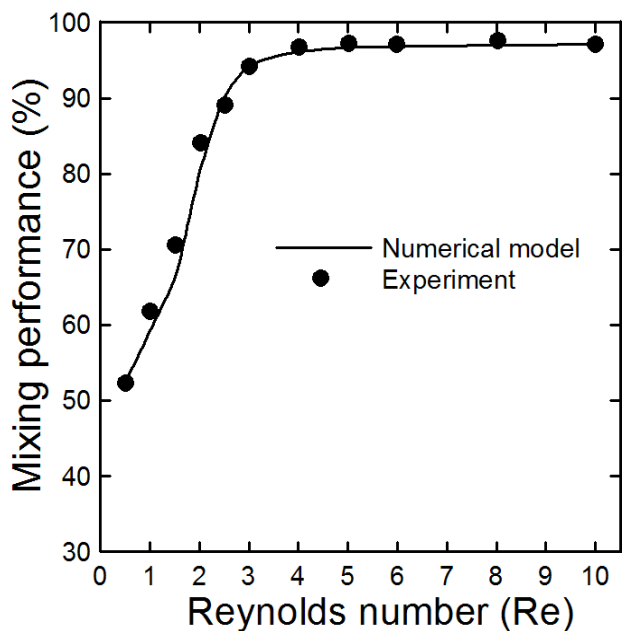

Figure 5. Micro-chamber mixing ratio given Reynolds numbers in the range of $\operatorname{Re}=0.5 \sim 10$.

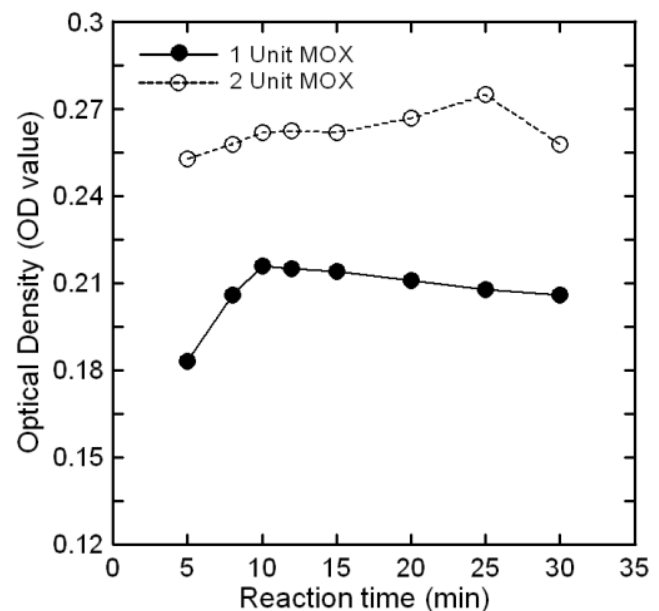

Figure 6. Minimum time required for different units of MOX with corresponding reaction times.

Figure 6 presents the optimized operating conditions for the proposed methanol detection system under different units of MOX with corresponding reaction times, at a methanol concentration of $30 \mathrm{ppm}$ and $\mathrm{Re}=4$. The result indicates that a higher unit MOX (that is, the two-unit MOX in Figure 6) can result in a faster reaction (approximately $10 \mathrm{~min}$ ), as well as an optical density suitable for the MOX-methanol reaction. Figure 7(a) presents the detected methanol concentration results using a traditional large-scale 

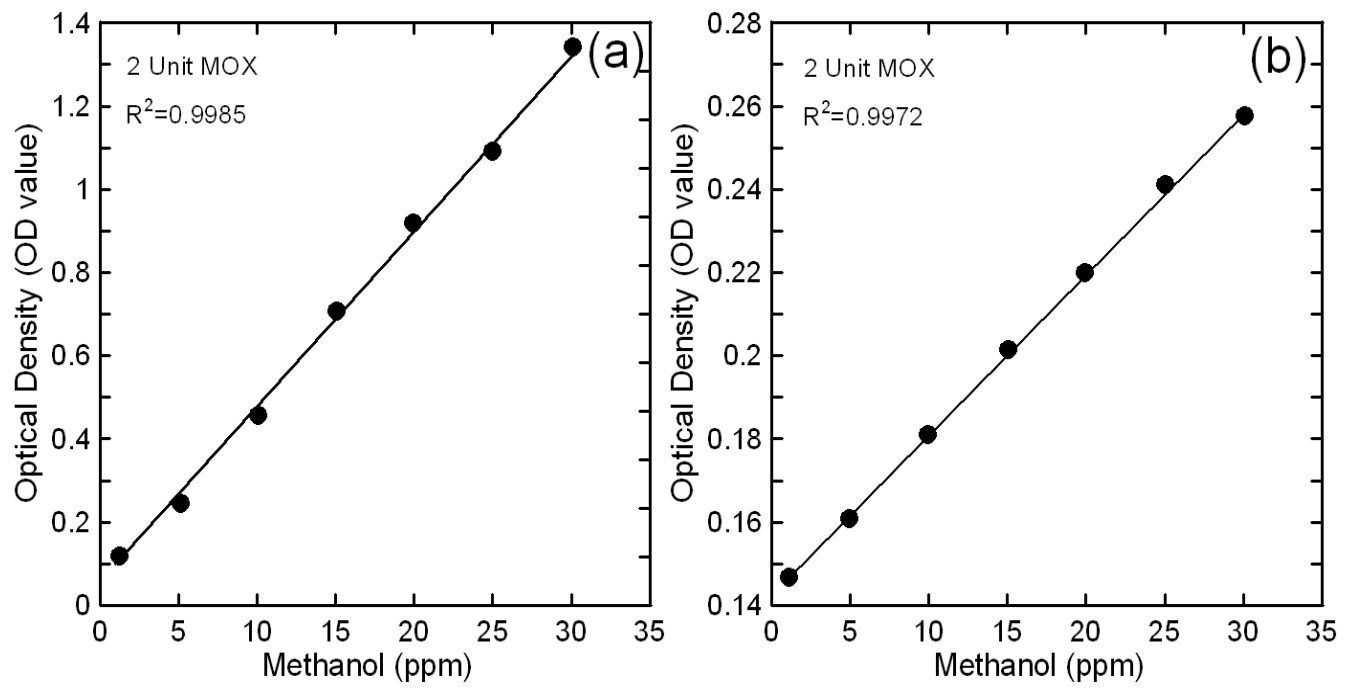

Figure 7. OD value of methanol detection results for (a) a conventional tube system and (b) an integrated microfluidic system.

system for methanol samples, with concentrations ranging from $10^{\sim} 30 \mathrm{ppm}$. The final methanol reactant was detected using a conventional UV spectrophotometer to detect the optical density (OD) value. The $R$ square and relationship were determined to be $R^{2}=0.9985$ and $Y=0.0581 X-0.4641$, respectively. Figure $7(b)$ presents the detected methanol concentration results using the proposed integrated microfluidic system for methanol samples with concentrations ranging from $10 \sim 30 \mathrm{ppm}$. The $\mathrm{R}$ square and relationship were determined to be $R^{2}=0.9972$ and $Y=0.0081 X-0.0442$, respectively. In this instance, a rapid and thorough mixing was achieved in the mixing and reaction column of the proposed microfluidic chip, such that $30 \mathrm{~min}$ of reaction time was adequate for completing methanol detection. Thus the proposed integrated microfluidic system is able to obtain a larger range of the methanol concentration detection. The result confirms that the proposed integrated microfluidic system is an effective, accurate, and rapid method for methanol detection.

\section{Conclusion}

This paper presents a low-cost, time-saving, and high-performance integrated microfluidic system for detecting methanol concentrations. The microchip is fabricated on a polymethyl-methacrylate (PMMA) substrate, thus providing advantages including easy fabrication, favorable biocompatibility, and excellent light transmission characteristics. An experimental platform was created for multiple reaction processes. The optimal microchannel geometry design miniaturizes the pressure drop and the uniform flow rate completes the methanol and enzyme reaction to form formaldehyde. Using a distinct microchip size for various UV spectrophotometers, the proposed detection method can provide a low-cost alternative to expensive quartz detection chips. A series of experimental tests compared the performance of the traditional concentration detection method and the proposed integrated microfluidic system concentration detection method for methanol samples with concentrations ranging from 10 30 ppm. The correlation coefficient obtained when plotting the absorbance against the methanol concentration had a value of $R^{2}=0.9985$ for the traditional detection method, and $R^{2}=0.9972$ for the integrated microfluidic detection method. The proposed rapid integrated methanol detection microchip is a post-processing chip. After combining the proposed microchip with various analyses for liquor, alcohol, synthetic alcohol, industrial alcohol, and methanol, the proposed integrated detection method will be used to develop a microchip to separate methanol.

\section{Acknowledgement}

The authors would like to thank Taiwan's National Science Council for providing financial support for this research.

\section{References}

[1] M. Koklu, S. Park, S. D. Pillai, and A. Beskok, "Negative dielectrophoretic capture of bacterial spores in food matrices," Biomicrofluidics, vol. 4, no. 3, p. 034107, 2010. doi: $10.1063 / 1.3479998$

[2] X. Weng, C. H. Chon, H. Jiang, and D. Li, "Rapid detection of formaldehyde concentration in food on a polydimethylsiloxane (PDMS) microfluidic chip," Food Chemistry, vol. 114, no. 3, pp. 1079-1082, 2009. doi: 10.1016/i.foodchem.2008.10.027 
[3] R. L. Smith, C. J. Demers, and S. D. Collins, "Microfluidic device for the combinatorial application and maintenance of dynamically imposed diffusional gradients," Microfluidics and Nanofluidics, vol. 9, no. 4-5, pp. 613-622, 2010. doi: 10.1007/s10404-010-0574-7

[4] R. Pethig, "Review article-dielectrophoresis: Status of the theory, technology, and applications," Biomicrofluidics, vol. 4, no. 2, p. 022811, 2010. doi: $10.1063 / 1.3456626$

[5] H. Chen and Z. H. Fan, "Two-dimensional protein separation in microfluidic devices," ELECTROPHORESIS, vol. 30, no. 5, pp. 758-765, 2009. doi: $10.1002 /$ elps.200800566

[6] M. Wang and Q. Kang, "Electrochemomechanical energy conversion efficiency in silica nanochannels," Microfluidics and Nanofluidics, vol. 9, no. 2-3, pp. 181-190, 2009. doi: 10.1007/s10404-009-0530-6

[7] K. Jang, Y. Xu, Y. Tanaka, K. Sato, K. Mawatari, T. Konno, K. Ishihara, and T. Kitamori, "Single-cell attachment and culture method using a photochemical reaction in a closed microfluidic system," Biomicrofluidics, vol. 4, no. 3, p. 032208, 2010. doi: $\underline{10.1063 / 1.3494287}$

[8] S. A. Vanapalli, M. H. G. Duits, and F. Mugele, "Microfluidics as a functional tool for cell mechanics," Biomicrofluidics, vol. 3, no. 1, p. 012006, 2009.

doi: $10.1063 / 1.3067820$

[9] S. Chiavaroli, D. Newport, and B. Woulfe, "An optical counting technique with vertical hydrodynamic focusing for biological cells," Biomicrofluidics, vol. 4, no. 2, p. 024110, 2010. doi: $\underline{10.1063 / 1.3380598}$

[10] S. N. Jayasinghe and N. Suter, "Pressure driven spinning: A multifaceted approach for preparing nanoscaled functionalized fibers, scaffolds, and membranes with advanced materials," Biomicrofluidics, vol. 4, no. 1, p. 014106, 2010. doi: $10.1063 / 1.3328092$

[11] X. Xuan, J. Zhu, and C. Church, "Particle focusing in microfluidic devices," Microfluidics and Nanofluidics, vol. 9, no. 1, pp. 1-16, 2010. doi: $10.1007 / \mathrm{s} 10404-010-0602-7$

[12] C. H. Tsai, H. H. Hou, and L. M. Fu, "An optimal three-dimensional focusing technique for micro-flow cytometers," Microfluidics and Nanofluidics, vol. 5, no. 6, pp. 827-836, 2008. doi: $10.1007 / \mathrm{s} 10404-008-0284-6$

[13] S. Balasuriya, "Optimal frequency for microfluidic mixing across a fluid interface," Physical Review Letters, vol. 105, no. 6, p. 064501, 2010. doi: $10.1103 /$ PhysRevLett.105.064501

[14] H. W. Wu, R. C. Hsu, C. C. Lin, S. M. Hwang, and G. B. Lee, "An integrated microfluidic system for isolation, counting, and sorting of hematopoietic stem cells,"
Biomicrofluidics, vol. 4, no. 2, p. 024112, 2010. doi: $10.1063 / 1.3454767$

[15] H. H. Hou, C. H. Tsai, L. M. Fu, and R. J. Yang, "Experimental and numerical investigation into micro-flow cytometer with 3-D hydrodynamic focusing effect and micro-weir structure," ELECTROPHORESIS, vol. 30, no. 14, pp. 2507-2515, 2009. doi: $10.1002 /$ elps.200900012

[16] L. M. Fu, R. J. Yang, C. H. Lin, Y. J. Pan, and G. B. Lee, "Electrokinetically driven micro flow cytometers with integrated fiber optics for on-line cell/particle detection," Analytica Chimica Acta, vol. 507, no. 1, pp. 163-169, 2004.

doi: $10.1016 /$ j.aca.2003.10.028

[17] M. Rosenauer and M. J. Vellekoop, "Characterization of a microflow cytometer with an integrated three-dimensional optofluidic lens system," Biomicrofluidics, vol. 4, no. 4, p. 043005, 2010. doi: $10.1063 / 1.3502672$

[18] H. Sun, Z. Nie, and Y. S. Fung, "Determination of free bilirubin and its binding capacity by hsa using a microfluidic chip-capillary electrophoresis device with a multi-segment circular-ferrofluid-driven micromixing injection," ELECTROPHORESIS, vol. 31, no. 18, pp. 3061-3069, 2010. doi: $10.1002 /$ elps.200900749

[19] C. Y. Lee, C. M. Chen, G. L. Chang, C. H. Lin, and L. M. $\mathrm{Fu}$, "Fabrication and characterization of semicircular detection electrodes for contactless conductivity detector - ce microchips," ELECTROPHORESIS, vol. 27, no. 24, pp. 5043-5050, 2006. doi: $10.1002 /$ elps.200600113

[20] L. M. Fu, C. Y. Lee, M. H. Liao, and C. H. Lin, "Fabrication and testing of high-performance detection sensor for capillary electrophoresis microchips," Biomedical Microdevices, vol. 10, no. 1, pp. 73-80, 2007. doi: $10.1007 / \mathrm{s} 10544-007-9111-1$

[21] Y. Lin, G. J. Gerfen, D. L. Rousseau, and S. R. Yeh, "Ultrafast microfluidic mixer and freeze-quenching device," Anal. Chem., vol. 75, no. 20, pp. 5381-5386, 2003. doi: $10.1021 /$ ac0346205

[22] L. M. Fu and C. H. Lin, "A rapid DNA digestion system," Biomedical Microdevices, vol. 9, no. 2, pp. 277-286, 2006. doi: $10.1007 / \mathrm{s} 10544-006-9036-0$

[23] C. Plaziac, P. Lachapelle, and C. Casanova, "Effects of methanol on the retinal function of juvenile rats," NeuroToxicology, vol. 24, no. 2, pp. 255-260, 2003. doi: 10.1016/S0161-813X(02)00165-1

[24] J. H. Wang, C. H. Wang, C. C. Lin, H. Y. Lei, and G. B. Lee, "An integrated microfluidic system for counting of CD4+/CD8+ T lymphocytes," Microfluidics and Nanofluidics, vol. 10, no. 3, pp. 531-541, 2010. doi: $10.1007 / \mathrm{s} 10404-010-0687-z$ 
[25] M. C. Wu, C. M. Jiang, Y. Y. Ho, S. C. Shen, and H. M. Chang, "Convenient quantification of methanol in juices by methanol oxidase in combination with basic fuchsin," Food Chemistry, vol. 100, no. 1, pp. 412-418, 2007.

doi: $10.1016 /$ j.foodchem.2005.09.017

[26] H. Puchtler, S. N. Meloan, and B. R. Brewton, "On structural formulas of basic fuchsin and aldehyde-schiff reaction products," Histochemistry, vol. 45, no. 4, pp. 255-265, 1975.

doi: $10.1007 / B F 00492627$
[27] T. F. Hong, W. J. Ju, M. C. Wu, C. H. Tai, C. H. Tsai, and L. M. Fu, "Rapid prototyping of PMMA microfluidic chips utilizing a co2 laser," Microfluidics and Nanofluidics, vol. 9, no. 6, pp. 1125-1133, 2010. doi: $10.1007 / \mathrm{s} 10404-010-0633-0$

[28] C. H. Lin, C. H. Tsai, C. W. Pan, and L. M. Fu, "Rapid circular microfluidic mixer utilizing unbalanced driving force," Biomedical Microdevices, vol. 9, no. 1, pp. 43-50, 2006. doi: $10.1007 / \mathrm{s} 10544-006-9009-3$ 